\title{
Clampe de Ganz no tratamento de urgência em lesões do anel pélvico*
}

\author{
Ganz clamps to treat emergencies in pelvic ring lesions
}

\author{
Gilberto José Cação Pereira ${ }^{1}$, Hamilton da Rosa Pereira', Daniel Innocenti Dinhani², \\ DAVI NICOLETTI GUMIEIRO'2, REINALDO dOS SANTOS VOLPI ${ }^{3}$
}

\section{RESUMO}

Objetivo: Avaliar a eficiência do clampe de Ganz na estabilização e redução dos deslocamentos da pelve, quando utilizado no tratamento de urgência, além de aquilatar eventuais dificuldades e facilidades do método. Métodos: O clampe de Ganz foi utilizado no tratamento de urgência em 31 pacientes com graves lesões do anel pélvico (Tile $\mathrm{C}$ ) associadas a importante instabilidade hemodinâmica. $R e$ sultado: Entre os pacientes, $27(87,1 \%)$ apresentaram evolução favorável, com estabilização, redução dos deslocamentos e compressão da região posterior do anel, além de estabilização hemodinâmica,

* Trabalho realizado no Departamento de Cirurgia e Ortopedia da Faculdade de Medicina de Botucatu da Universidade Estadual Paulista "Júlio de Mesquita Filho" - UNESP - Botucatu (SP), Brasil.

1. Doutor, Professor Assistente do Departamento de Cirurgia e Ortopedia da Faculdade de Medicina de Botucatu da Universidade Estadual Paulista "Júlio de Mesquita Filho" - UNESP - Botucatu (SP), Brasil.

2. Médico do Departamento de Cirurgia e Ortopedia da Faculdade de Medicina de Botucatu da Universidade Estadual Paulista "Júlio de Mesquita Filho" - UNESP - Botucatu (SP), Brasil.

3. Doutor, Médico do Departamento de Cirurgia e Ortopedia da Faculdade de Medicina de Botucatu da Universidade Estadual Paulista "Júlio de Mesquita Filho" - UNESP - Botucatu (SP), Brasil.

Endereço para correspondência: Prof. Dr. Gilberto José Cação Pereira, Departamento de Cirurgia e Ortopedia - Faculdade de Medicina de Botucatu - Unesp, Campus de Rubião Júnior - 18618-970 Botucatu (SP), Brasil. Tel.: (14) 3811-6230, fax: (14) 3815-7615. E-mail: gpereira@fmb.unesp.br

Recebido em 17/4/08. Aprovado para publicação em 24/6/08. Copyright RBO2008 e quatro $(12,9 \%)$ evoluíram para óbito. Conclusão: O clampe de Ganz mostrou-se eficiente ferramenta no tratamento de urgência das lesões do anel pélvico, por ser de concepção simples, de rápida colocação, não impedir ou dificultar procedimentos no abdome e, principalmente, por permitir a estabilização da pelve, redução dos deslocamentos e compressão na região posterior do anel, local onde ocorrem os maiores sangramentos, reduzindo-os ou eliminando-os.
Descritores - Articulação sacroilíaca/lesões; Ossos pélvi- cos/lesões; Hemodinâmica; Hemorragia; Emergências

\section{ABSTRACT}

Objective: To evaluate the effectiveness of Ganz clamp to stabilize and reduce pelvic dislocations in emergency situations, and to assess possible difficult and easy aspects of the method. Method: Ganz clamp was used in the emergency treatment of 31 patients with severe lesions of the pelvic ring (Tile $C$ ) associated to major hemodynamic instability. Results: Among the patients, 27 (87.1\%) presented a favorable evolution, with stabilization, reduction of dislocations, and compression of the posterior portion of the ring, and also hemodynamic stabilization, and $4(12.9 \%)$ died. Conclusion: The Ganz clamp was found to be an effective tool in the emergency treatment of pelvic ring lesions because it has an uncomplicated conception, allowing for a fast placement, because it does not prevent nor make it difficult for abdominal procedures, and most of all because it allows for the stabilization of the pelvis, 
reduction of dislocations, and compression in the posterior region of the ring, where most bleeding occurs, to reduce or eliminate bleeding.

Keywords - Sacroiliac joint/injuries; Pelvic bones/
injuries; Hemodynamics; Hemorrhage;
Emergencies

\section{INTRODUÇÃO}

Os traumas de alta energia, frequientes em acidentes automobilísticos, de motocicletas ou queda de grandes alturas, podem provocar importantes lesões do anel pélvico, com graves consequiências. Dentre essas lesões, as que envolvem a região posterior do anel encontram-se entre as mais sérias, particularmente as disjunções completas das articulações sacroilíacas, associadas às fraturas do púbis e ísquio, ou à disjunção da sínfise púbica, pois estão sujeitas a grandes deslocamentos e, conseqüentemente, graves lesões vasculares.

Segundo a literatura, a mortalidade em lesões do anel pélvico varia de 8 a $50 \%$ e a causa mais freqüente é a grande perda sanguínea ${ }^{(1-6)}$. Estudos clínicos e anatômicos desse tipo de lesão têm demonstrado que o sangramento venoso difuso é o mais frequiente e, em torno de $20 \%$ dos casos, o sangramento é arterial e, nesses, as artérias glúteas são as mais lesadas ${ }^{(7-12)}$.

O objetivo do tratamento de urgência nestas graves lesões do anel pélvico é a redução e estabilização desse anel, com intuito de diminuir espaços e impedir movimentos, buscando-se assim conter o sangramento. Entre os métodos que podem ser utilizados na tentativa de contenção do sangramento está a "vestimenta" pneumática antichoque (PASG) que, por meio da compressão das extremidades, promove a redistribuição do volume sanguíneo e, pela compressão do abdome e região pélvica, pode limitar a expansão do hematoma e estabilizar o anel. O método apresenta um inconveniente, que é a freqüente necessidade de suporte ventilatório, devido à compressão da região do abdome ${ }^{(8-}$ ${ }^{9,13)}$. O fixador externo anterior é freqüentemente utilizado no tratamento de emergência para a estabilização e contenção do sangramento em lesões do anel pélvico, mas tem ação limitada na região posterior do anel, local onde ocorrem as maiores perdas sanguíneas ${ }^{14-}$ 15).

O dispositivo desenvolvido por Ganz et al ou clampe pélvico de Ganz é um dos métodos indicados no tratamento de urgência das lesões do anel pélvico, pois estabiliza e proporciona compressão na região posterior do anel, local mais crítico e importante da lesão(15-17).

Os casos de lesões graves do anel pélvico que tratamos na urgência, com o fixador externo anterior, não apresentaram boa estabilização da região posterior do anel nem adequada redução dos deslocamentos nesse local, o que dificultou a contensão do sangramento. Decidimos, assim, avaliar as facilidades ou dificuldades do método e, principalmente, a eficiência do clampe na estabilização e diminuição do espaço na região posterior do anel.

\section{MÉTODOS}

O clampe pélvico de Ganz foi utilizado, no Departamento de Cirurgia e Ortopedia da Faculdade de Medicina de Botucatu, em 31 pacientes com lesões do anel pélvico, sendo $26(83,8 \%)$ do sexo masculino e cinco $(16,2 \%)$ do feminino. A idade variou entre $10 \mathrm{e}$ 54 anos (28 adultos e três crianças) (figura 1). As causas das lesões foram acidentes automobilísticos em 23 $(74,2 \%)$, com motocicleta em cinco $(16,1 \%)$ e três $(9,6 \%)$ com trator ou máquinas industriais. Nos acidentes automobilísticos, 21 pacientes encontravam-se no veículo e dois foram vítimas de atropelamento. Foram incluídos no estudo somente sérias lesões do anel (tipo Tile $\mathrm{C}^{(11)}$ ) que apresentavam instabilidade hemodinâmica, mesmo já tendo recebido em torno de $1.500-2.000 \mathrm{ml}$ de reposição líquida (Ringer-lactato, sangue) no Setor de Emergência. Entre os pacientes, 27 apresentaram uma ou mais lesões concomitantes: fraturas, laceração hepática, pneumotórax, hemotórax, ruptura vesical e trauma craniano.

Para a colocação do clampe foi utilizado o método preconizado por Ganz et al (figura 2)(15). Inicialmente, é identificado o ponto de penetração do pino de fixação no ilíaco, que corresponde a um ponto localizado a aproximadamente $8-10 \mathrm{~cm}$ ântero-lateral à espinha ilíaca póstero-superior, ao longo de uma linha imaginária entre esta e a espinha ilíaca ântero-superior (fi- 

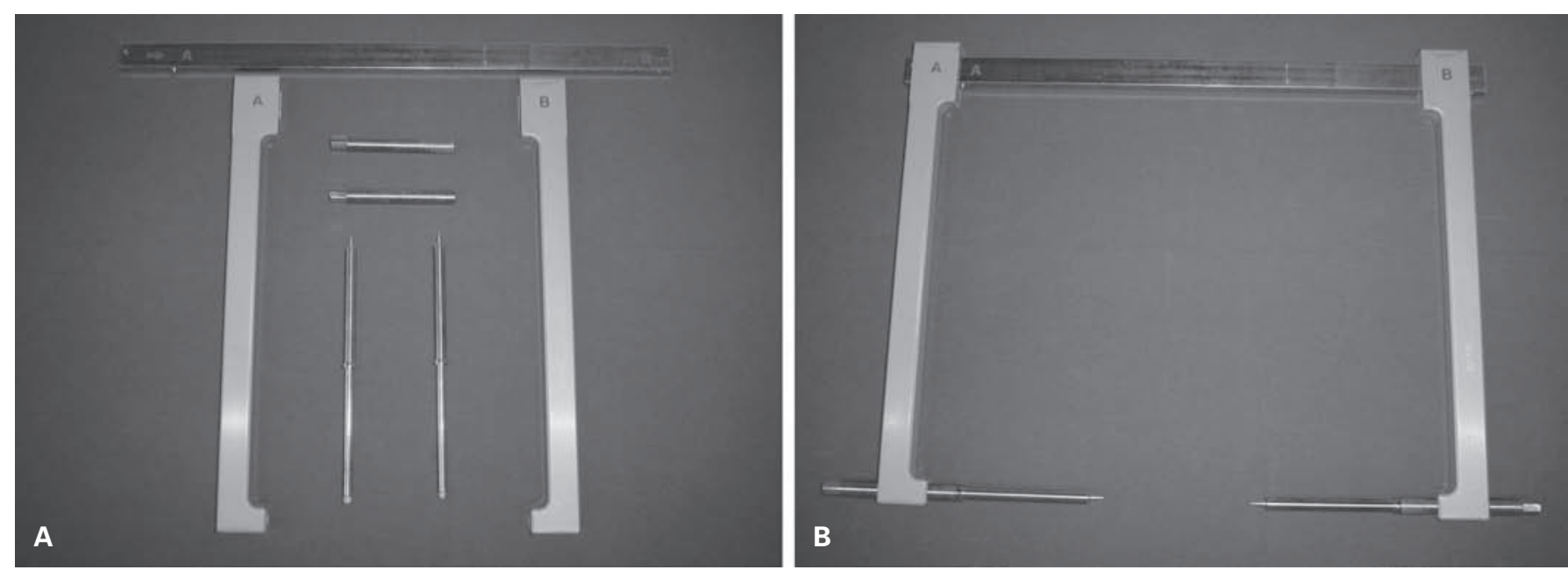

Figura 1 - Componentes do clampe de Ganz (A); clampe montado (B).
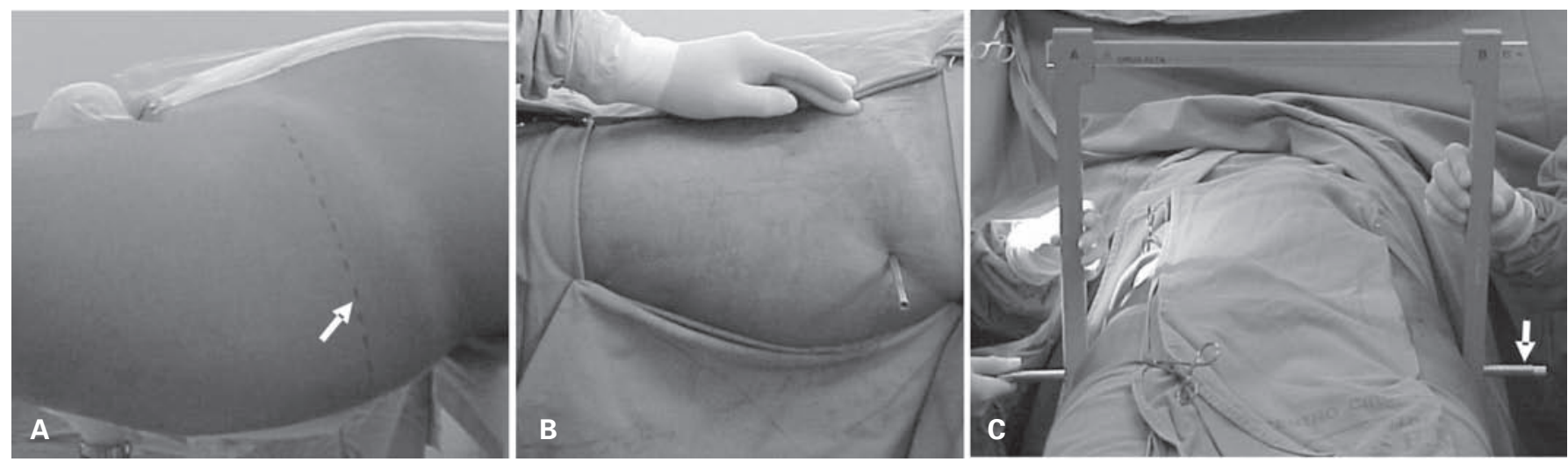

Figura 2 - Linha imaginária entre a espinha ilíaca ântero-superior e a espinha ilíaca ântero-posterior com a seta indicando o ponto onde o pino deve ser introduzido (A); localização da espinha ilíaca ântero-superior e o pino já fixado no ilíaco (B); clampe de Ganz montado e fixado aos pinos, a seta indica o parafuso onde se realiza a compressão.

gura 2A). O pino é introduzido através de pequena incisão e sua ponta é fixada ao osso ilíaco com auxílio de um martelo (figura 2B). O procedimento é realizado em ambos os lados da pélvis. Após a fixação dos pinos, os outros componentes do clampe são acoplados a estes e realiza-se a impacção do anel, por meio do parafuso de compressão (figura 2C). Em todos os casos o clampe foi mantido de três a cinco dias (média de três dias) e após a retirada foi realizada a fixação definitiva do anel pélvico.

\section{RESULTADOS}

Dos casos estudados, $27(87,1 \%)$ apresentaram boa evolução, com imediata redução dos deslocamentos e rígida fixação da região posterior do anel pélvico, além de estabilização hemodinâmica, que ocorreu em aproximadamente 40 minutos após a colocação do clampe. Nos casos em que o clampe permaneceu por cinco dias, houve infecção no local de penetração do pino de fixação, resolvida rapidamente após a retirada do mesmo e limpeza local, e quatro casos $(12,9 \%)$ evoluíram para óbito.

\section{DISCUSSÃO}

As lesões do anel pélvico, principalmente as que envolvem a articulação sacroilíaca, são consideradas como importantes, pois, dependendo da proporção do deslocamento, podem estar associadas a graves lesões 


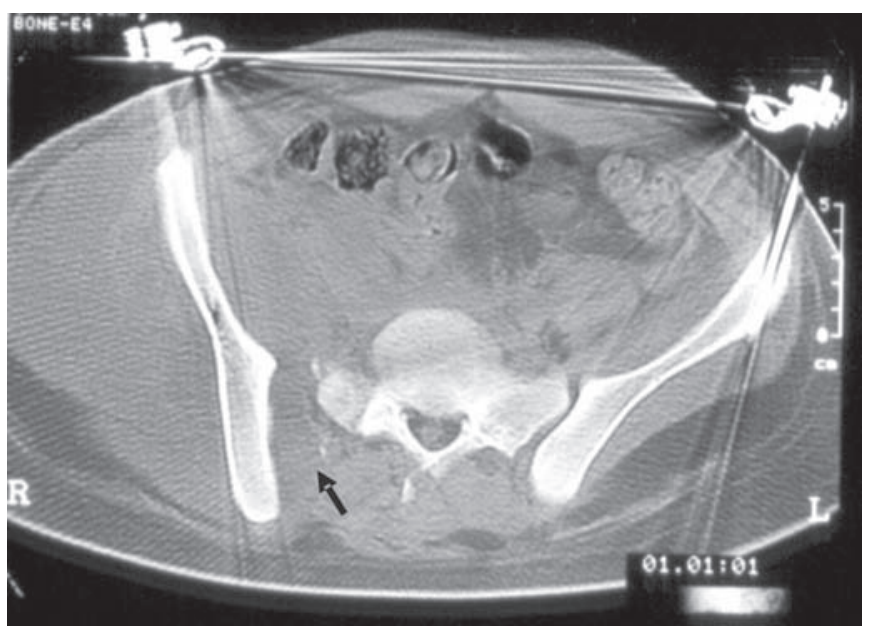

Figura 3 - Tomografia mostrando uma imagem do anel pélvico com um fixador externo anterior e a seta indicando a abertura posterior

do plexo venoso pré-sacral ${ }^{(15-16)}$. Apesar dos maiores cuidados que ultimamente se tem dispensado a esses pacientes e das tentativas de melhora no atendimento desse tipo de trauma, a literatura relata índices de mortalidade que variam entre 6 e $27 \%$, geralmente por grande perda sanguínea, sendo mais elevados se forem analisados somente os casos de graves lesões do anel pélvico, associadas à instabilidade hemodinâmi$\mathrm{ca}^{(1,3,18-19)}$. Mucha et al, em análise sobre o tratamento de fraturas pélvicas, observaram que nos casos de lesões associadas à instabilidade hemodinâmica, as quais denominou "complicadas", o índice de mortalidade foi de $42 \%{ }^{(20)}$.

A fixação externa tem sido considerada o método mais efetivo para a estabilização do anel pélvico e controle do sangramento venoso difuso ${ }^{(18,21)}$. O fixador externo anterior, colocado rapidamente, pode diminuir o sangramento e melhorar os resultados clínicos; no entanto, é limitado na estabilização do anel pélvico e não permite compressão da região posterior, justamente onde ocorrem as maiores perdas sanguíneas ${ }^{(16,22)}$. Além disso, dependendo da compressão anterior que se realiza no fixador, pode aumentar a abertura na região posterior do anel, dificultando ainda mais a contenção do sangramento (figura 3).

O clampe pélvico promove compressão justamente na região posterior do anel, local mais importante da

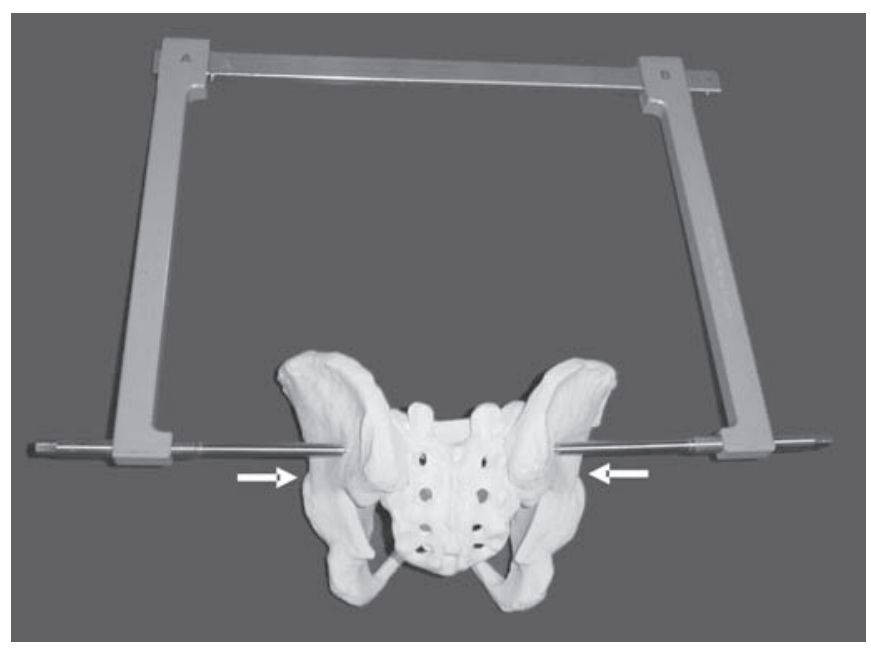

Figura 4 - Visão posterior do anel pélvico fixado com o clampe de Ganz. As setas indicam o local e direção da compressão.

lesão e onde ocorrem os grandes sangramentos (figura 4).

Huittinen et al, em estudo angiográfico pós-morte em pacientes com lesões do anel pélvico, referem que a região sacroilíaca é uma área crucial nesse tipo de trauma, sendo o local onde foram observados os maiores hematomas ${ }^{(10)}$. Simonian et al, visando avaliar a estabilização do anel, realizaram um trabalho biomecânico comparativo entre o fixador externo anterior e o clampe pélvico; concluíram que a região posterior é melhor estabilizada pelo clampe, pois este sistema permite menor grau de movimentação no local ${ }^{(16)}$. Tile refere que, em lesões verticalmente instáveis, o fixador externo anterior não estabiliza de maneira rígida a região posterior do anel ${ }^{(11)}$. O clampe pélvico é considerado um fixador externo da parte posterior do anel. Possui desenho simples, pode ser instalado rapidamente, não exige necessariamente sala cirúrgica para sua colocação e, pelo fato de ser móvel, não impede o acesso ao abdome ou à região pélvica. Em três dos nossos casos, a fixação foi realizada na própria sala de emergência. Além disso, quando foi necessária intervenção na região abdominal ou pélvica, não houve qualquer dificuldade, pois a barra transversa pode ser deslocada no sentido cranial ou caudal (figura 5).

Quanto à técnica de colocação do clampe, apesar da simplicidade do dispositivo, nos primeiros casos tive- 

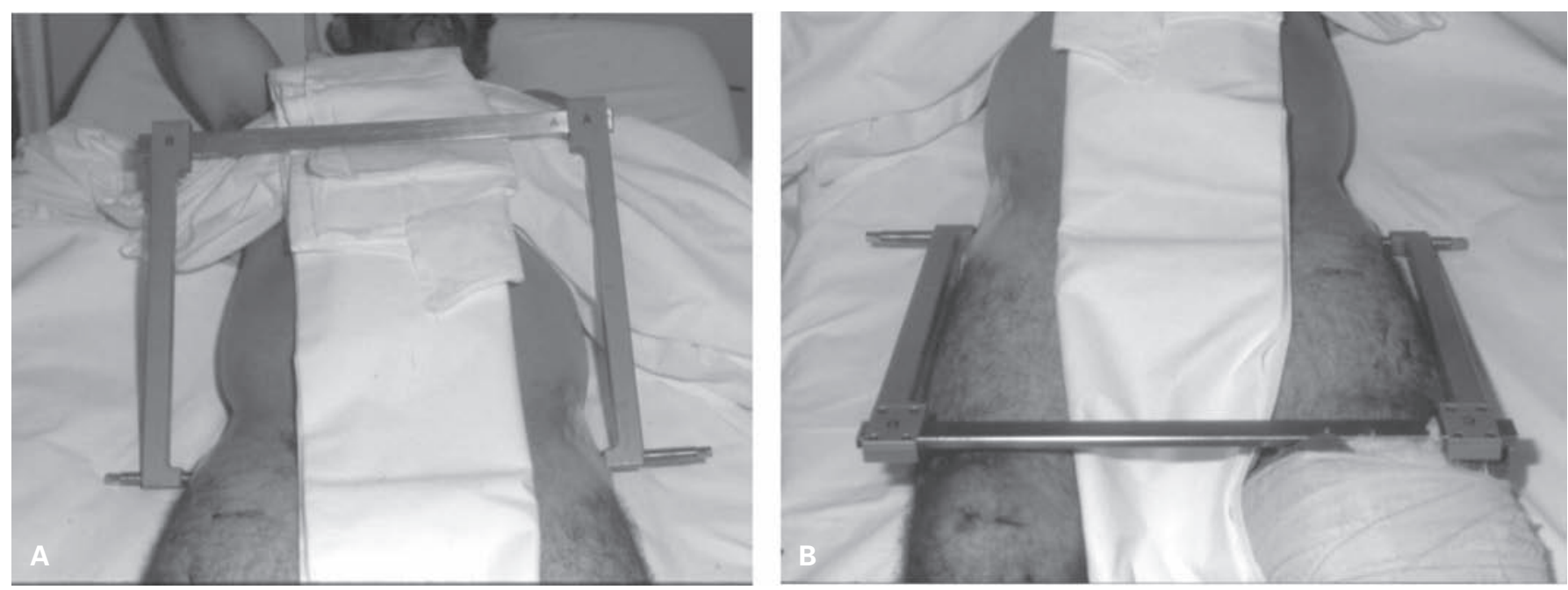

Figura 5 - O clampe de Ganz pode ser deslocado no sentido cranial (A) e caudal (B)

mos certa dificuldade na localização do melhor ponto para a fixação no ilíaco, principalmente nos pacientes obesos ou com importante edema e hematoma na região do quadril, o que dificultava a palpação dos pontos de referência. Essa dificuldade foi gradativamente diminuindo à medida que fomos adquirindo maior experiência com a técnica; no entanto, em alguns desses casos, também utilizamos o intensificador de imagem e consideramos que este auxilia muito na localização do ponto de fixação no ilíaco. Tivemos uma única intercorrência com a utilização do clampe: um dos pacientes, com grande abertura da sacroilíaca, apresentava grande ruptura do retroperitônio, através da qual houve protrusão de uma alça intestinal; um segmento dela alojou-se na sacroilíaca, sendo comprimido no momento em que o clampe foi apertado. Pela particularidade do caso, julgamos que foi uma rara intercorrência, não encontrando descrição semelhante na literatura.

O clampe de Ganz é indicado em lesões instáveis do anel pélvico, isto é, disjunções importantes da articulação sacroilíaca, associadas a significante perda sanguínea. No entanto, não está indicado em casos com grave fragmentação óssea, próximo à articulação sacroilíaca, pois não será efetivo e seus pinos poderão penetrar através da fratura e causar lesões neurológicas, vasculares ou viscerais ${ }^{(15-16)}$.

Todos os nossos casos submetidos à fixação com o clampe pélvico apresentavam graves lesões do anel pélvico e importante instabilidade hemodinâmica, apesar dos procedimentos de rotina para politraumatizados (ATLS), tais como: reposição volumétrica em torno de $1.500-2.000 \mathrm{ml}$ e tratamento de lesões concomitantes. Dos 31 pacientes submetidos à fixação com o clampe pélvico, $27(87,1 \%)$ apresentaram boa evolução, redução dos deslocamentos e rígida fixação da sacroilíaca, com reconstituição imediata da estrutura anatômica do anel (figura 6). Em associação ocorreram: melhora gradativa dos níveis pressóricos e estabilização hemodinâmica, aproximadamente 30 a 40 minutos após a fixação, mostrando ser o método efetivo no tratamento de urgência desse tipo de lesão.

Em um dos nossos pacientes, tomado como exemplo, após a colocação do clampe, observou-se a reconstituição da estrutura anatômica do anel pélvico, com fechamento da sínfise púbica e da sacroilíaca direita (figuras 7 e 8). Por meio da ficha anestésica observa-se que no início da anestesia (X) os níveis pressóricos ( $\vee$ ) estavam em torno de 9,5-6,5 e a freqüência cardíaca ( ), em torno de 95 batimentos, apesar de já ter sido realizada reposição volumétrica $(2.000 \mathrm{ml})$ no setor de emergência. No início da fixação $(\phi)$, os níveis pressóricos já haviam caído, atingindo 9,0-4,0, mesmo tendo sido administrado volume adicional de Ringer-lactato $(\nabla)$. Durante a colocação do clampe, apesar de o paciente ter recebido mais volume líqui- 

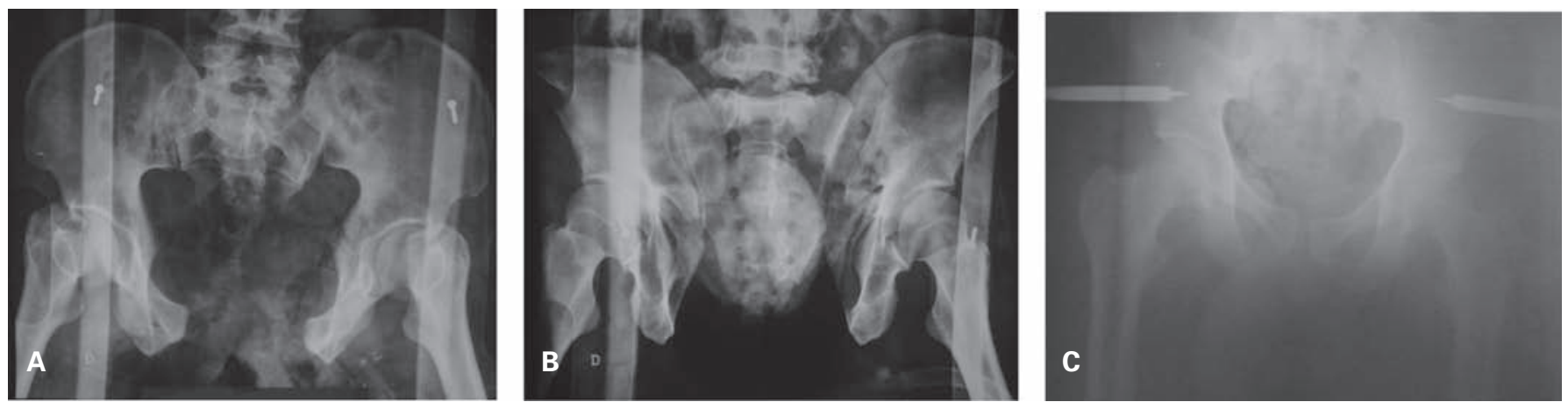

Figura 6 - Na incidência AP, observa-se grande abertura da sínfise púbica e sacroilíaca esquerda (A). Na projeção out-let, também é identificada abertura da sacroilíaca e hemiascensão à esquerda (B). O controle radiológico pós-fixação mostra o fechamento da sínfise púbica e da sacroilíaca, com reconstituição anatômica do anel (C).

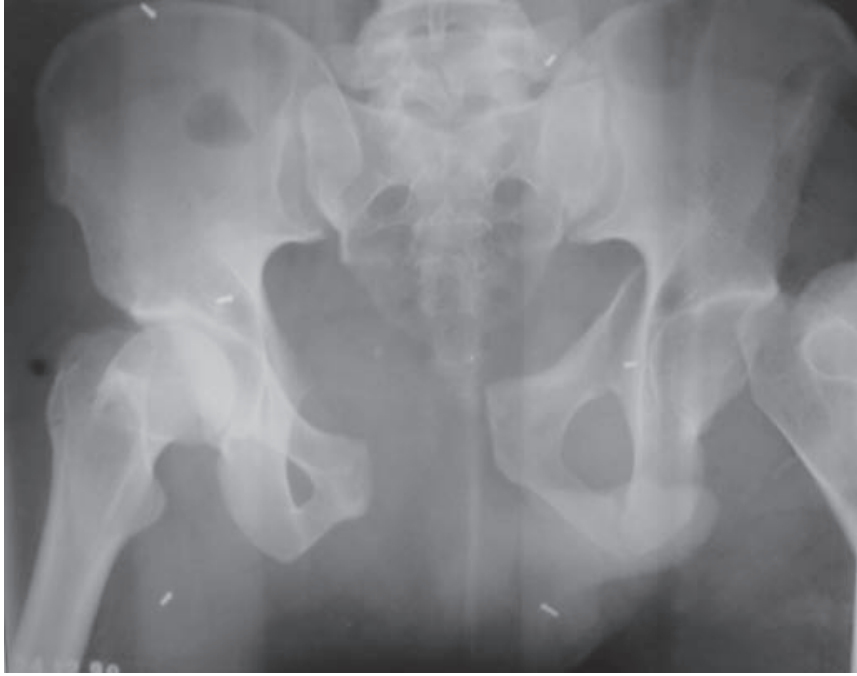

Figura 7 - 0 exame radiológico mostra abertura da sínfise púbica, fratura do ísquio e púbis, disjunção da sacroilíaca direita e luxação coxofemoral à esquerda

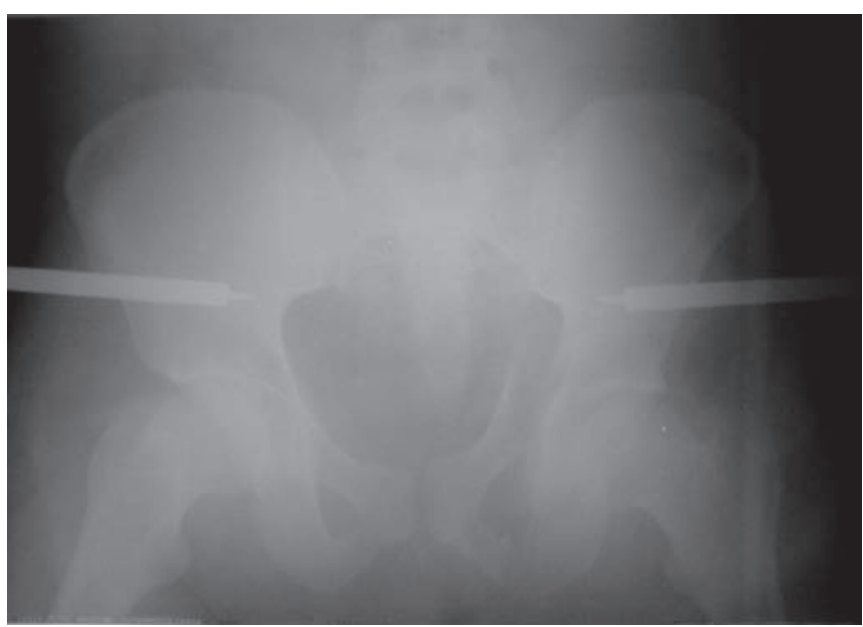

Figura 8 - Radiografia de controle após a redução da luxação e colocação do clampe

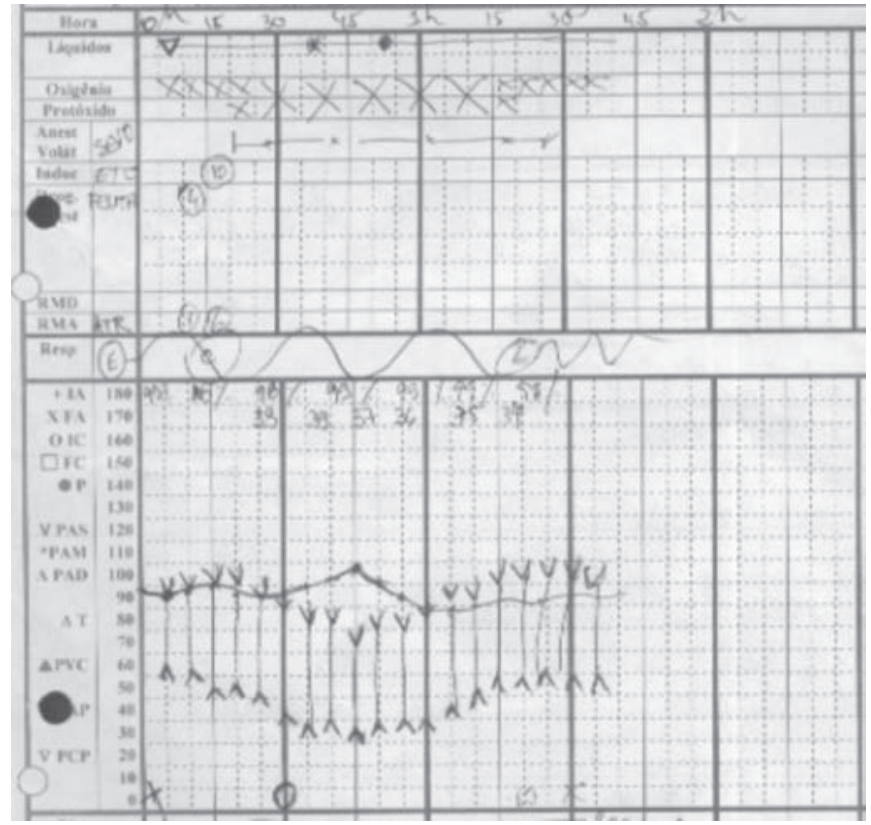

Figura 9 - Ficha anestésica: início da anestesia (X), nível pressórico $(\vee)$, freqüência cardíaca $(\sim)$, início da fixação $(\phi)$, Ringer-lactato $(\nabla)$ volume $\left({ }^{*}\right)$

do, os níveis pressóricos diminuíram ainda mais, chegando a atingir 7,5-3,5 e a frequiência cardíaca, 110 . Em torno de 30 minutos após o início da fixação, os níveis pressóricos começaram a se elevar e a freqüência cardíaca diminuir, ocorrendo em seguida estabilização da pressão em torno de 10,0-6,0 e a freqüência cardíaca, em 90 batimentos, sem administração adicional de líquidos (figura 9).

Tiemann et al, também utilizando o clampe pélvico em pacientes com graves lesões do anel, referem que em parte deles a pressão sanguínea se elevou 20 minu- 

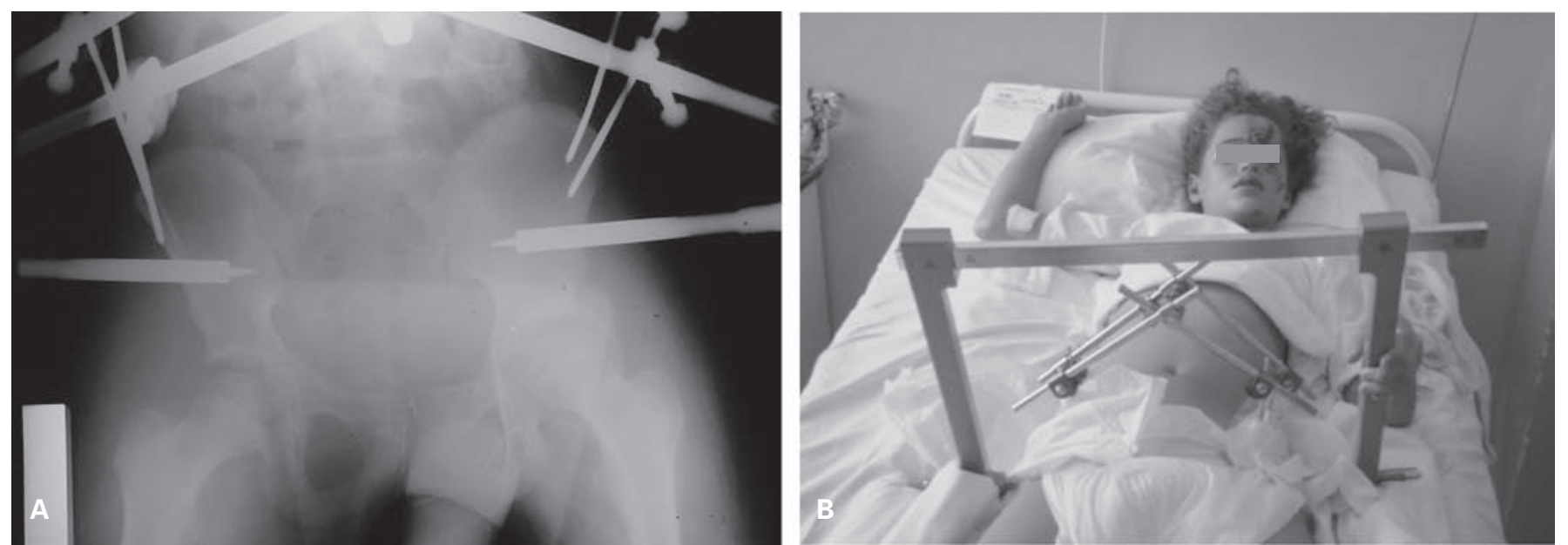

Figura 10 - Exame radiológico mostrando o clampe de Ganz associado ao fixador externo anterior (A); fotografia do paciente (B).

tos após a colocação do clampe e, em seguida, houve estabilização hemodinâmica ${ }^{(21)}$.

Em um dos nossos pacientes (criança vítima de atropelamento), a fixação externa anterior foi associada ao clampe pélvico, pois com isso conseguimos a manutenção mais adequada da estrutura anatômica anterior do anel (figura 10). Holt et al referem que a lesão do anel pélvico é pouco freqüente em crianças, mas também nessa faixa etária o clampe de Ganz é método apropriado para o tratamento da lesão, quando associado à instabilidade hemodinâmica, pois proporciona estabilização dos níveis pressóricos, não causa lesão da cartilagem do ilíaco e permite acesso ao abdome e pelve ${ }^{(23)}$.

Em relação ao período de permanência da fixação, Ganz et al referem que ainda não existe definição quanto ao tempo que o clampe pode ser mantido no local, sem risco de infecção ${ }^{(15)}$. Na maioria dos nossos casos, o clampe foi mantido em torno de três dias até que o paciente apresentasse condições cirúrgicas para a fixação definitiva. Nos casos em que o clampe permaneceu por períodos mais prolongados, houve sinais de infecção no local de penetração dos pinos, que regrediu rapidamente após a retirada do material e limpeza local. Em um dos casos, no segundo dia pós-fixação, houve soltura do clampe pela excessiva manipulação do paciente, mas não houve problema, pois ele já se encontrava hemodinamicamente estável. Em quatro dos nossos pacientes, o sangramento pélvico não foi contido mesmo com a compressão e redução da região posterior do anel, obtidas com a colocação do clampe; a perda sanguínea foi considerada causa importante do óbito. Não foi identificada lesão em grandes vasos na região pélvica, mas houve considerável sangramento, apesar da embolização e exploração cirúrgica com tamponamento pélvico. A embolização e tamponamento pélvico são procedimentos recomendados em casos de lesões instáveis do anel pélvico, quando a fixação externa não é suficiente para a estabilização hemodinâmica e não são identificados outros locais de perda sanguínea; no entanto, mesmo essas medidas extremas podem não conter o sangramento ${ }^{(17,24)}$. Os pacientes que foram a óbito também apresentavam lesões associadas (laceração hepática, hemotórax e lesão vesical), que certamente agravaram a perda sanguínea e contribuíram para evolução desfavorável desses casos.

\section{CONCLUSÃO}

O clampe de Ganz mostrou-se eficiente ferramenta no tratamento de urgência das lesões do anel pélvico, por ser de concepção simples, de rápida colocação, não impedir ou dificultar procedimentos no abdome e, principalmente, por permitir a estabilização da pelve, redução dos deslocamentos e compressão na região posterior do anel, local onde ocorrem os maiores sangramentos, reduzindo-os ou eliminando-os. 


\section{REFERÊNCIAS}

1. Fangio P, Asehnoune K, Edouard A, Smail N, Benhamou D. Early embolization and vasopressor administration for management of life-threatening hemorrhage from pelvic fracture. J Trauma. 2005;58(5):978-84; discussion 984.

2. Ertel W, Keel M, Eid K, Platz A, Trentz O. Control of severe hemorrhage using C-clampe and pelvic packing in multiply injured patients with pelvic ring disruption. J Orthop Trauma. 2001;15(7):468-74. Comment in: J Orthop Trauma. 2002; 16(5):362; author reply 362-3.

3. Katsoulis E, Drakoulakis E, Giannoudis P. Management of open pelvic fractures. Curr Orthop. 2005;19(5):345-53.

4. Moreno C, Moore EE, Rosenberger A, Cleveland HC. Hemorrhage associated with major pelvic fracture: a multispecialty challenge. J Trauma. 1986;26(11):987-94.

5. Patterson FP, Morton KS. The cause of death in fractures of the pelvis: with a note on treatment by ligation of the hipogastric (internal iliac) artery. J Trauma. 1973;13(10):849-56.

6. Rothenberger D, Velasco R, Strate R, Fischer RP, Perry JF Jr. Open pelvic fracture: a lethal injury. J Trauma. 1978;18(3): 184-7.

7. Browner BD, Cole JD, Graham JM, Bondurant FJ, NunchuckBurns SK, Colter HB. Delayed posterior internal fixation of unstable pelvic fractures. J Trauma. 1987;27(9):998-1006.

8. Flint LM Jr, Brown A, Richardson JD, Polk HC. Definitive control of bleeding from severe pelvic fractures. Ann Surg. 1979;189(6):709-16.

9. Gilliland MG, Ward RE, Flynn TC, Miller PW, Ben-Menachem Y, Duke JH Jr. Peritoneal lavage and angiography in the management of patients with pelvic fractures. Am J Surg. 1982; 144(6):744-7.

10. Huittinen VM, Slätis P. Postmortem angiography and dissection of the hypogastric artery in pelvic fractures. Surgery. 1973;73(3):454-62.

11. Tile M. Fractures of the pelvis and acetabulum. $2^{\text {nd }}$ ed. Baltimore: Williams \& Wilkins; 1995. p. 66-149.

12. Yellin AE, Lundell CJ, Finck EJ. Diagnosis and control of posttraumatic pelvic hemorrhage: transcatheter angiographic embolization techniques. Arch Surg. 1983;118(12):1378-83.
13. Batalden DJ, Wichstrom PH, Ruiz E, Gustilo RB. Value of the $\mathrm{G}$ suit in patients with severe pelvic fracture. Controlling hemorrhagic shock. Arch Surg 1974;109(2):326-8.

14. Dahners LE, Jacobs RR, Jayraman G, Cepulo AJ. A study of external skeletal fixation systems for unstable pelvic fractures. J Trauma. 1984;24(10):876-81.

15. Ganz R, Krushell RJ, Jakob RP, Küffer J. The antishock pelvic clamp. Clin Orthop Relat Res. 1991;(267):71-8.

16. Simonian PT, Routt ML Jr, Harrington RM, Tencer AF. Anterior versus posterior provisional fixation in the unstable pelvis - A biomechanical comparison. Clin Orthop Relat Res. 1995;(310):245-51.

17. Sadri H, Nguyen-Tang T, Stern R, Hoffmeyer P, Peter R. Control of severe hemorrhage using C-clamp and arterial embolization in hemodynamically unstable patients with pelvic ring disruption. Arch Orthop Trauma Surg. 2005;125(7):443-7.

18. Gylling SF, Ward RE, Holcroft JW, Bray TJ, Chapman MW. Immediate external fixation of unstable pelvic fractures. Am J Surg. 1985;150(6):721-4.

19. Pohlemann T, Braune C, Gänsslen A, Hüfner T, Partenheimer A. Pelvic emergency clamps: anatomic landmarks for a safe primary application. J Orthop Trauma. 2004;18(2):102-5.

20. Mucha P Jr, Farnell MB. Analysis of pelvic fracture management. J Trauma. 1984;24(5):379-86.

21. Tiemann AH, Schmidt C, Gonschorek O, Josten C. [Use of the "c-clamp" in the emergency treatment of unstable pelvic fractures]. Zentralbl Chir. 2004;129(4):245-51. German.

22. Edwards CC, Meier PJ, Browner BD, Freedman MA, Ackley SM. Results treating 50 unstable pelvic injuries using primary external fixation. Orthop Trans. 1985;9:434-9.

23. Holt GE, Mencio GA. Pelvic C-clamp in a pediatric patient. J Orthop Trauma. 2003;17(7):525-7.

24. Ruchholtz S, Waydhas C, Lewan U, Pehle B, Taeger G, Kühne C, Nast-Kolb D. Free abdominal fluid on ultrasound in instable pelvic ring fracture: is laparotomy always necessary? J Trauma. 2004;57(2):278-85; discussion 285-7. 